1 Universidade de Brasília (UnB), Programa de Pós-Graduação em Saúde Coletiva - Brasília (DF), Brasil.

emanuellyps@hotmail.com

2 Universidade de Brasília (UnB), Programa de Pós-Graduação em Saúde Coletiva -Brasília (DF), Brasil.

magscherer@hotmail.com

3 Fundação Oswaldo Cruz (Fiocruz), Escola Nacional de Saúde Pública Sérgio Arouca (Ensp), Centro de Saúde Escola Germano Sinval Farias - Rio de Janeiro (RJ), Brasil. odwyer@ensp.fiocruz. com.br

\section{Inserção de um hospital de grande porte na Rede de Urgências e Emergências da região Centro-Oeste}

\author{
Inclusion of a large size hospital in the Urgency and Emergency \\ Network of the Center-West region
}

Emanuelly Paulino Soares', Magda Duarte dos Anjos Scherer², Gisele O'Dwyer ${ }^{\mathbf{3}}$
RESUMO Em face da exigência de se configurar as redes de cuidados, este estudo qualitativo analisa a inserção de um hospital de grande porte na Rede de Atenção às Urgências e Emergências na região Centro-Oeste do Brasil utilizando dados secundários, análise documental e entrevistas semiestruturadas aplicadas aos gestores e profissionais que atuam no Centro de Trauma. Os resultados sugerem desconhecimento dos profissionais quanto à inserção do Hospital na rede; definição de fluxos e pactos incipiente; boas práticas na regulação pelo Serviço de Atendimento Móvel de Urgências. Conclui-se que a inserção do Hospital acontece de forma lenta e depende da articulação dos vários níveis do Sistema Único de Saúde.

PALAVRAS-CHAVE Assistência à saúde; Serviços de saúde; Emergências.

ABSTRACT In view of the demand of configuring the care networks, this qualitative study analyses the insertion of a large size hospital in Urgent and Emergency Care Network in the CenterWest region of Brazil based on secondary data, documentary analysis and semi-structured interviews applied to managers and professionals that work in the Hospital's Trauma Center. The results suggest unawareness of the professionals on the insertion of the Hospital in the network; flows definitions and incipient pacts; good practices in regulation by the Emergency Mobile Care Service. It is concluded that the insertion of the Hospital takes place slowly and depends on the interaction of multi-level of the Unified Health System.

KEYWORDS Assistance to health; Health services; Emergencies. 


\section{Introdução}

Mudanças no cenário mundial apontam desafios para a saúde pública nos contextos epidemiológico e sociodemográfico atuais, especialmente nos países em desenvolvimento. O envelhecimento da população, a partir do aumento da expectativa de vida e da consequente queda das taxas de natalidade, é um dos fatores preocupantes, bem como o número crescente de pessoas acometidas por doenças e agravos mais comuns, em que as causas externas ocupam posição de destaque, especialmente os acidentes de trânsito e as violências, além das doenças do aparelho circulatório, com destaque para as doenças cardiovasculares e cerebrovasculares.

Embora sejam reconhecidos os avanços do Sistema Único de Saúde (SUS), como inovação institucional no campo das políticas sociais (MAIO; LIMA, 2009), ao longo dos seus 25 anos, um dos grandes problemas para a sua consolidação tem sido o modelo de atenção e gestão predominantemente fragmentado e não coincidente com as necessidades de saúde geradas pela transição sociodemográfica e epidemiológica.

Nesse contexto, a implementação de redes de atenção à saúde se torna fundamental. Com essa perspectiva, o Ministério da Saúde (MS) publicou, em 2010, as diretrizes para a organização da Rede de Atenção à Saúde (RAS), no âmbito do SUS. Esse normativo pode ser considerado um marco histórico no processo de organização da assistência à saúde nos estados e municípios, com ênfase na organização dos territórios em regiões de saúde, resgatando o contexto histórico que justifica a implantação, bem como conceitos, fundamentos, elementos e estratégias para constituição da RAS (BRASIL, 2010).

Do mesmo modo, em 2011, o MS instituiu a Rede de Atenção às Urgências e Emergências (RUE) como estratégia fundamental para a consolidação do SUS, de modo a promover e assegurar a universalidade e a integralidade da atenção, a equidade do acesso, além de transparência na alocação de recursos dos serviços e ações de saúde no âmbito da atenção às situações de urgência e emergência (BRASIL, 2011A).

Desde as primeiras normas sobre o tema urgência/emergência, foi proposta pelo MS a utilização do termo 'urgência' em substituição dos distintos conceitos de urgência e emergência, onde, segundo a Resolução CFM no 1451/95, a 'emergência' exige atendimento no nível hospitalar e a 'urgência' é compatível com os níveis de atenção primária e secundária.

As primeiras portarias que compuseram a política de urgência trataram dos componentes pré-hospitalares, com destaque para o Serviço de Atendimento Móvel de Urgência (Samu) e as Unidades de Pronto Atendimento (UPA). No mesmo ano em que foi instituída a RUE, o MS apresentou o arcabouço normativo do seu Componente Atenção Hospitalar, com o objetivo de ampliar e qualificar as portas de entrada hospitalares de urgência, as enfermarias clínicas de retaguarda, as enfermarias de retaguarda de longa permanência e os leitos de terapia intensiva e de reorganizar as linhas de cuidados prioritárias de traumatologia, cardiovascular e cerebrovascular (BRASIL, 2011B), enfrentando, entre outros, o problema crônico da superlotação. A superlotação nas emergências aumenta o risco de mortalidade em casos atendidos com atraso e causa descontentamento nos demandantes, independentemente da gravidade do caso. Outra consequência é a flexibilização dos padrões de cuidado e da ética dos profissionais de saúde que atuam na urgência (O'DWYER, 2010).

O enfrentamento das situações de urgências e emergências e de suas causas requer não apenas a assistência imediata, mas ações de promoção da saúde e prevenção de doenças e agravos, tratamento contínuo das doenças crônicas, reabilitação e cuidados paliativos.

A RUE deve ser implementada de forma gradativa, em todo o território nacional, 
respeitando critérios epidemiológicos e de densidade populacional. A organização da RUE se justifica pela necessidade de articular e integrar todos os equipamentos de saúde, objetivando ampliar e qualificar o acesso humanizado e integral dos usuários em situação de urgência e emergência nos serviços de saúde, de forma ágil e oportuna (BRASIL, 2013). A implantação dessa rede de cuidados é estratégia potente para a consolidação do SUS no Brasil.

O hospital, na rede de atenção, é referência em determinadas situações de maior complexidade ou gravidade, contribuindo para a integralidade do cuidado ao fazer adequada contrarreferência após realizar o atendimento (CECILIO; MERHY, 2003).

Para compreender a inserção de um hospital de grande porte na rede, deve-se considerar o arcabouço normativo que orienta a RUE no SUS e a atividade concreta que envolve o trabalho 'vivo' dos gestores e trabalhadores da saúde na sua relação com o meio, como geradores de mudança e mobilização. Este artigo tem por objetivo analisar a inserção de um hospital de grande porte na RUE na região Centro-Oeste.

\section{Método}

Trata-se de um estudo descritivo com abordagem qualitativa (MINAYO, 2008), desenvolvido num hospital de grande porte da região Centro-Oeste do Brasil, de julho a agosto de 2014, tendo como cenário o serviço de pronto-socorro e Centro de Trauma do Hospital.

A investigação foi guiada pelas seguintes indagações: Como se insere um hospital na RUE? Como são aplicados os critérios de qualificação do componente Atenção Hospitalar da RUE, à luz das diretrizes estabelecidas pelo MS no serviço de pronto-socorro deste Hospital?

Este estudo foi iniciado com a caracterização do Hospital, utilizando dados gerais apresentados no Sistema de Cadastro Nacional de Estabelecimentos de Saúde (SCNES) (BRASIL, 2014A) e informações do sistema de registros de atendimentos do Hospital. Na sequência, foram realizadas entrevistas semiestruturadas com gestores e profissionais de saúde responsáveis pelos pacientes graves que chegam até o pronto-socorro, Centro de Trauma do Hospital: três médicos, quatro enfermeiras, três técnicos de enfermagem e três gestores, totalizando 13 participantes. O roteiro continha questões que buscavam traçar um breve perfil dos participantes, sobre a organização do serviço de pronto-socorro e o processo de inserção do Hospital na RUE - dirigido a todos os participantes - e sobre a aplicação dos critérios de qualificação do serviço de pronto-socorro, de acordo com normativos do MS - dirigido apenas aos profissionais da assistência.

Para preservar o anonimato dos participantes, principalmente dos gestores, que estão em pequeno número, a identidade do Hospital não é revelada. Os profissionais são identificados pela letra $\mathrm{P}$ e os gestores pela letra G, seguidos da ordem com que o questionário foi respondido.

Os profissionais de saúde que participaram do estudo tinham idade entre 25 e 48 anos, quatro eram pós-graduados, cinco graduados e um tinha cursado o ensino médio. Com relação ao vínculo empregatício, oito eram concursados e dois contratados. Dos dez profissionais, sete informaram que realizam cursos de aperfeiçoamento em suas áreas de atuação. Os gestores tinham idade entre 33 e 42 anos, todos pós-graduados e concursados. Apenas um gestor não possuía experiência anterior na gestão.

Os resultados foram organizados em três categorias: Breve caracterização do cenário da pesquisa; Inserção do Hospital na Rede de Atenção às Urgências e Emergências; Processo de qualificação do serviço de pronto-socorro a partir dos critérios estabelecidos pelo MS. 
O estudo foi aprovado junto ao Comitê de Ética e Pesquisa da Universidade de Brasília, sob No 705.216, em julho de 2014.

\section{Resultados e discussão}

\section{Breve caracterização do cenário da pesquisa}

\section{SOBRE O HOSPITAL}

O Hospital é classificado como hospital especializado tipo $\mathrm{II}^{\mathbf{1}}$ e de ensino, justificado pela obtenção de habilitações como centro de referência nas áreas de alta complexidade cardiovascular (pediátrica, vascular e intervencionista), nefrologia, neurologia, neurocirurgia, oncologia, Aids, cuidados prolongados (enfermidades cardiovasculares, pneumológicas, oncológicas, neurológicas e provocadas por causas externas), internação domiciliar, terapia enteral e parenteral, transplantes (córnea, rim e retirada de órgãos e tecidos) e trauma-ortopedia (BRASIL, 2014A). Essa última refere-se à missão mais prioritária e fundamental para a rede de atenção às urgências e emergências, por ser referência na prestação de cuidados na área e para a região.

No que diz respeito à gestão, possui gestão estadual, esfera administrativa também estadual e natureza de organização direta. Presta atividades ambulatoriais e hospitalares de média e alta complexidades. Em junho de 2014, possuía 855 leitos, sendo 819 destinados ao atendimento no SUS, o que representa mais de $90 \%$ de sua capacidade total. Esses leitos se subdividem em 464 leitos cirúrgicos, 299 clínicos, 29 de terapia intensiva e 56 de pediatria clínica e cirúrgica (BRASIL, 2014B).

\section{SOBRE O SERVIÇO DE PRONTO-SOCORRO, CENTRO DE TRAUMA DO HOSPITAL}

O serviço de pronto-socorro do Hospital está dividido em três clínicas de atendimento às urgências e emergências: pediatria, cérebro e cardiovascular e trauma. Na descrição de um dos profissionais

O pronto-socorro deste Hospital é ímpar por se tratar de um hospital terciário, logo, tendo como função a realização de diagnóstico, exames e tratamentos de alta complexidade. Dessa forma, o paciente do pronto-socorro é, em geral, de alta complexidade, se tratando de doentes graves que exigem profissionais treinados e qualificados. Por ser referência em trauma, essa complexidade do pronto-socorro apenas aumenta. (P1).

$\mathrm{O}$ número de atendimentos registrados no serviço de pronto-socorro, Centro de Trauma do Hospital, é crescente se observarmos os registros de atendimento mensal durante um ano. No ano de 2013, o mês de setembro se destaca com o maior número de atendimentos. Percebeu-se, ainda, que os casos por doenças e agravos relacionados a causas externas, especialmente traumas e violências, têm uma forte relação com a questão de gênero, considerando que a maior parte refere-se à entrada de homens, superior ao dobro da de mulheres (tabela 1).

O número de atendimentos por procedência Centro de Trauma - Sala Vermelha do Hospital, no ano de 2013, está expresso na (tabela 2).

\footnotetext{
1 Hospital especializado, tipo II, destinado à prestação de assistência à saúde na modalidade de internação em uma ou mais especialidades, excetuando-se clínica médica e cirurgia geral, dispondo de Serviço de Atendimento Diagnóstico e Terapêutico (SADT), podendo contar com serviço de Urgência/ Emergência, Unidade de Terapia Intensiva, hospitaldia, serviço ambulatorial e outros. O tipo corresponde ao número de habilitações de serviços estratégicos para urgência e emergência no Hospital, a exemplo de neurocirurgia, cardiovascular ou traumatologia (BRASIL,
} 2013). 


\begin{tabular}{lllllllllllllll}
\hline \multicolumn{10}{l}{ Tabela 1. Atendimentos realizados na Sala Vermelha do Centro de Trauma do Hospital, 2013} \\
\hline \multicolumn{10}{c}{ ATENDIMENTOS NA SALA VERMELHA POR GÊNERO } \\
\hline JAN & FEV & MAR & ABR & MAI & JUN & JUL & AGO & SET & OUT & NOV & DEZ \\
\hline HOMENS & 94 & 120 & 107 & 88 & 122 & 107 & 105 & 121 & 128 & 131 & 108 & 108 \\
MULHERES & 44 & 33 & 38 & 30 & 32 & 31 & 34 & 40 & 48 & 41 & 48 & 55 \\
TOTAL & $\mathbf{1 3 8}$ & $\mathbf{1 5 3}$ & $\mathbf{1 4 5}$ & $\mathbf{1 1 8}$ & $\mathbf{1 5 4}$ & $\mathbf{1 3 8}$ & $\mathbf{1 3 9}$ & $\mathbf{1 6 1}$ & $\mathbf{1 7 6}$ & $\mathbf{1 7 2}$ & $\mathbf{1 5 6}$ & $\mathbf{1 6 3}$ \\
\hline
\end{tabular}

Nota: Elaboração própria

Tabela 2. Atendimentos realizados por procedência dos pacientes na Sala Vermelha do Centro de Trauma do Hospital, 2013

\begin{tabular}{lcccccccccccc}
\hline & \multicolumn{1}{c}{ PROCEDÊNCIA DOS PACIENTES } & & & \\
& JAN & FEV & MAR & ABR & MAI & JUN & JUL & AGO & SET & OUT & NOV & DEZ \\
\hline INTRA-HOSPITALAR & 30 & 19 & 23 & 20 & 44 & 25 & 28 & 33 & 40 & 33 & 34 & 45 \\
EXTRA-HOSPITALAR & 108 & 134 & 122 & 98 & 110 & 113 & 111 & 128 & 136 & 139 & 122 & 118 \\
TOTAL & $\mathbf{1 3 8}$ & $\mathbf{1 5 3}$ & $\mathbf{1 4 5}$ & $\mathbf{1 1 8}$ & $\mathbf{1 5 4}$ & $\mathbf{1 3 8}$ & $\mathbf{1 3 9}$ & $\mathbf{1 6 1}$ & $\mathbf{1 7 6}$ & $\mathbf{1 7 2}$ & $\mathbf{1 5 6}$ & $\mathbf{1 6 3}$ \\
\hline
\end{tabular}

Nota: Elaboração própria

A maior parte dos atendimentos é dirigida aos pacientes que são encaminhados pelos serviços de saúde da rede assistencial dos estados da região Centro-Oeste ou é fruto de demanda espontânea, que chega ao Hospital sem nenhum encaminhamento ou com encaminhamentos não regulados.

Com relação às características $d a$ demanda, com base em levantamentos diários dos atendimentos, os gestores fazem os seguintes apontamentos: "Alta taxa de internação na Unidade de Neurocirurgia, pois somos a única referência na região" (G1). "Urgência trauma, IAM [infarto agudo do miocárdio] e $A V C$ [acidente vascular cerebral] e geralmente não referenciados" (G2). "Complexa e variável" (G3).

Ao discorrer sobre a situação do pronto-socorro, os profissionais destacam algumas dificuldades:

[...] a presença dos profissionais antigos pouco ativos e não abertos a mudanças e melhorias; dificuldade no acesso de medicamentos e exames de baixo custo; complexidade política em que o
Hospital está inserido; além da alta demanda. Outro problema é a vazão [saída] dos pacientes após os cuidados. (P1).

"Nem todas as equipes trabalham em harmonia [se referindo a possíveis problemas entre as equipes do pronto-socorro]" (P2). "[...] falta de material, de equipamentos, mínima estrutura" (P8).

[...] equipe rotativa demais; plantões com equipe deficiente; poucos funcionários; chefia pouco 'capacitada'; número excessivo de pacientes, que ficam 'internados' nas salas de entrada do servico esperando vagas em UTI, por exemplo; chefia pouco integrada com as equipes de entrada. (P7).

Muitas das dificuldades percebidas pelos participantes da pesquisa se assemelham ao que é relatado na literatura. Os serviços de emergência possuem como características inerentes: o acesso irrestrito; o número excessivo de pacientes; a extrema diversidade da gravidade no quadro inicial, tendo-se pacientes críticos ao lado de pacientes mais 
estáveis; a escassez de recursos; a sobrecarga da equipe de enfermagem; o número insuficiente de médicos; o predomínio de jovens profissionais; a fadiga; a supervisão inadequada; a descontinuidade do cuidado; e a falta de valorização dos profissionais envolvidos (SOUZA; SILVA; NORI, 2007).

No Brasil, parte expressiva da população busca consultas nos prontos-socorros dos hospitais, apesar da ampliação da oferta de serviços de Atenção Básica, desde 1990. Assim, os hospitais ainda são importante porta de entrada para a assistência médica, o que pode se relacionar às dificuldades no acesso oportuno a serviços básicos, especializados e de apoio diagnóstico. Ademais, o sentido de urgência para o paciente pode não ser o mesmo para profissionais de saúde (MACHADO; SALVADOR; O'DWYER, 2011), contribuindo para a grande demanda por esses serviços, que tem como causa essencial a incapacidade de oferta do SUS.

A efetivação do trabalho em rede é um desafio para o SUS, especialmente no âmbito da urgência e emergência. A resolutividade da Atenção Básica está associada à inclusão do atendimento à demanda espontânea e do primeiro atendimento às urgências, absorvendo, dessa forma, uma demanda que hoje se encontra nas portas de entrada hospitalares, quando estas deveriam se destinar ao atendimento de urgências mais graves ou emergências.

\section{A INSERÇÃO DO HOSPITAL NA REDE DE ATENÇÃO ÀS URGÊNCIAS E EMERGÊNCIAS}

Todos os profissionais participantes do estudo informaram que desconhecem o processo de inserção do Hospital na RUE e destacaram a importância de participarem da gestão e da tomada de decisão: "Estar informado das decisões da gestão e rotinas [que] estão em processo de implantação" (P4). "Quem trabalha na ponta problematiza melhor (P5). "[...] o P.S. [pronto-socorro] será o primeiro atendimento" ( $\mathrm{P} 8)$.
A centralidade do componente da ação humana em todo o processo do cuidado à saúde tem como consequência a importância do trabalho dos profissionais na qualidade da prestação de serviços. Sendo assim, as condições oferecidas para o desenvolvimento do trabalho, a motivação dos profissionais de saúde e suas inter-relações devem contribuir de forma significativa para a melhoria da qualidade da atenção à saúde (MENDES, 2013, P. 162).

Todos os gestores participantes da pesquisa consideram que o gestor tem papel chave no processo de inserção do Hospital na rede de atenção às urgências: "A gestão imediata incentiva servidores e traça metas" (G1). "Todos os servidores do pronto-socorro foram orientados da importância da RUE” (G2).

Entretanto, os profissionais de saúde que participaram do estudo informaram desconhecer o processo de implantação da RUE.

Os participantes do estudo atribuem como entraves ao processo de implantação da RUE a rotatividade e a centralidade hierárquica das decisões na gestão provocadas pelas mudanças políticas; o pouco interesse e envolvimento dos gestores e profissionais de saúde; a demanda de usuários acima do esperado; e os problemas na efetivação da referência e da contrarreferência entre os serviços: "[Falta de] interesse dos profissionais e [problemas gerados pela rotatividade e centralidade hierárquica das decisões na gestão] questões políticas”(P1). "[Pouco] envolvimento dos profissionais" (P6). "Superlotação e falta de preparo dos profissionais" (G1). "[Problemas na efetivação da] referência e contrarreferência". (G2).

Trabalhar na porta de entrada de um hospital público com emergência é ser interpelado por uma demanda à qual dificilmente se poderá responder. Esta, além do excessivo volume, não se esgota no que, stricto sensu, considera-se um problema de saúde e em muito ultrapassa o que os serviços de saúde estão geralmente organizados para reconhecer e intervir. A frase, muito repetida pelos profissionais, 'Tudo bate na porta 
de entrada', além de remeter aos problemas produzidos na própria dinâmica interna do Hospital, indica, principalmente, a enormidade e a diversidade da demanda que ali chega (SÁ; CARRETEIRO; FERNANDES, 2008).

Além das dificuldades, os participantes apontam perspectivas de melhoria no funcionamento do pronto-socorro e da RUE: sensibilização dos profissionais de saúde, provocando maior envolvimento e protagonismo; capacitação dos trabalhadores da urgência; maiores investimentos (infraestrutura, aquisição de equipamentos), incluindo contratação de trabalhadores e melhores salários; superação de problemas políticos (rotatividade, verticalização, autocracia); melhorias na rede de atenção à saúde, principalmente na comunicação entre os serviços de saúde, a partir de pacto estabelecido entre os gestores que aponte para fluxos assistenciais bem definidos, bem como qualificação no pronto-socorro no momento em que se recebe um usuário.

Os relatos a seguir confirmam o que foi descrito acima: "As regionais têm que funcionar. Este Hospital é terciário [não deve receber pacientes que exijam cuidados primários ou secundários]" (P5). "Mudança de pensamento dos profissionais; qualificação dos profissionais; maior investimento; melhores salários" (P1). "Definir rotinas, orientar usuários e servidores" (G1). "Após triagem, melhor remanejamento dos casos 'verde' e 'azuis' [sinalização do protocolo de Manchester, que se refere aos casos não graves e que deveriam ser resolvidos no âmbito da Atenção Básica ou ambulatorial especializada] para outro serviço" (P6).

Estudo realizado para conhecer os sentimentos de profissionais dos serviços de pronto-socorro infantil diante de situações de trabalho no município de Recife (PE), em 2003, nos ajuda a compreender a complexidade de ser trabalhador num serviço de urgência-emergência. Segundo Feliciano, Kovacs e Sarinho (2005, P. 320) ao traçar uma aproximação ao dia-a-dia dos hospitais de pronto-socorro infantil é possível encontrar um trabalho que entrelaça a responsabilidade com pacientes graves e seus familiares, a grande demanda, a insuficiência de recursos materiais e humanos, os baixos salários, os múltiplos empregos e consequente cansaço, motivando uma contínua tensão emocional. Nesse contexto, manifestam-se sentimentos (aspirações dirigidas à satisfação das necessidades) e desejos (percepções das possibilidades de satisfazer essas necessidades) que não expressam exclusivamente uma necessidade particular, visto que podem ser compartilhados por outros membros das equipes.

Quando questionados quanto às possibilidades de contribuição dos trabalhadores do serviço de pronto-socorro do Hospital, no tocante à efetivação da RUE e à superação de problemas, os profissionais de saúde do pronto-socorro destacaram: "Fundamental. Dele [profissional de saúde] depende o primeiro atendimento, o tempo de diagnóstico e o próprio diagnóstico e, consequentemente, $o$ tempo de início do tratamento" (P1). "O trabalhador vivencia os problemas, diferente de quem está de longe, somente na parte teórica" (P2). "É a base [profissional de saúde], é quem de fato irá colocar em prática, [sua participação é] essencial" (P7).

Os participantes do estudo são unânimes em reconhecer a importância de envolver os trabalhadores no processo de tomada de decisões, pois, se os resultados obtidos não agregam valor para a ação cotidiana de quem opera o trabalho (ou seja, os profissionais de saúde), este não impacta em mudanças para quem o consome (o usuário). Nesse sentido, faz-se necessário um processo de problematização da realidade, que, em geral, é disparado no monitoramento e na avaliação do serviço.

As práticas avaliativas têm sido cada vez mais estimuladas ao longo do processo de aprimoramento do sistema de saúde brasileiro, tornando-se gradativamente reconhecidas como uma atividade essencial no contexto de formulação e redirecionamento 
de programas e políticas de saúde. Desde que concebida em suas múltiplas dimensões (parceria, participação, negociação, julgamento e produção de conhecimento, entre outras), a avaliação pode e deve ser utilizada como instrumento de poder, em uma perspectiva dialógica, em sociedades éticas, com foco na justiça Social (DUBEUX; FREESE; FELISBERTO, 2013).

Processo de qualificação do serviço de pronto-socorro a partir dos critérios estabelecidos pelo Ministério da Saúde

As respostas dos profissionais de saúde participantes do estudo, no tocante à aplicação dos critérios estabelecidos pelo Ministério da Saúde, foram organizadas conforme roteiro estabelecido na própria norma.

Os participantes foram questionados quanto ao conhecimento e à adoção de protocolos clínicos no serviço de pronto-socorro do Hospital. Os seis profissionais que afirmaram utilizar protocolos clínicos trabalhavam na Sala Vermelha, indicando que essa é uma prática localizada em um setor e, portanto, não institucionalizada. Foi informado pelos participantes da pesquisa o uso de um conjunto de Protocolos Clínicos: $\mathrm{ACLS}^{2}, \mathrm{ATLS}^{3}, \mathrm{APH}$ fixo ${ }^{4}$, abdome agudo e trauma e rotinas de enfermagem.

Uma das perguntas do questionário dizia respeito ao conhecimento dos profissionais sobre o processo de acolhimento com classificação de risco. Dos nove respondentes, oito desconheciam quem se responsabilizava por coordenar essa atividade. A classificação de risco tem por objetivo orientar o atendimento por nível de gravidade e sofrimento, sob responsabilidade da equipe de enfermagem, além de priorizar o acolhimento de demandas.

O Acolhimento com a Avaliação e Classificação de Riscos é um dispositivo criado por uma política pública de humanização dos serviços de saúde, que traz no bojo de sua definição uma postura ética, que implica o compartilhamento de saberes e angústias entre profissionais e usuários. Toma para si a responsabilidade de acolher o outro em suas demandas, com responsabilidade e resolutividade. Não deve ser entendido como uma etapa em um processo, mas, sim, como uma ação que precisa ocorrer em todos os locais e momentos do serviço de saúde (DEMORI; PIZZOLATO, 2007).

A emergência do Hospital trabalha integrada com o Samu e o Corpo de Bombeiros, constituindo uma articulação em rede. Essa articulação foi reconhecida pelos profissionais de saúde, que identificaram o Centro de Trauma como parte do Samu no Hospital. "[Funciona], principalmente, no trauma pelo Samu e bombeiros" (P1). "O Samu funciona dentro da emergência” (P3).

Quanto à regulação de leitos no Hospital, houve dissenso entre os participantes, fato esse que pode demonstrar fragilidade no processo de regulação intra-hospitalar, da porta de entrada para a retaguarda, conforme justificativa a seguir, que aponta para superlotação na emergência e rede de cuidados sem suporte: "Seria necessário outro hospital para atender" (P1). "[...] Mas é meio complicado, pois são muitos pacientes” (P8).

As dificuldades na regulação vivenciadas não são exclusividade do Hospital estudado. Estudo realizado em hospitais regionais de Pernambuco, em 2012, chama atenção para a necessidade da qualificação dos equipamentos de saúde nos três níveis assistenciais e para os mecanismos de regulação, sob o ponto de vista da minimização e da organização da demanda à rede de atenção à urgência hospitalar, quer seja pelos eventos agudos decorrentes de doenças crônicas, quer seja pela resolubilidade de unidades pré-hospitalares (DUBEUX; FREESE; FELISBERTO, 2013).

A literatura sinaliza que a multiprofissionalidade no trabalho em saúde contribui para ampliar a qualidade da atenção à saúde, possibilitando a integração de vários olhares e práticas para atuar frente à complexidade do processo saúde-doença-cuidado (PEDUZZI, 2001). Todos os profissionais participantes do estudo informaram a existência de equipe multiprofissional atuando no serviço de pronto-socorro, Centro de
2 ACLS - Curso baseado em ciência e evidências médicas, desenvolvido pela American Heart.

(http://educacao.cardiol. br/cursos/acls.asp, 2014). 3 ATLS - Curso destinado à capacitação de trabalhadores médicos e enfermeiros que atuam no manejo direto aos agravos relacionados ao trauma (http://www.cets.com.br/ cursos_atls.php, 2014). 4 APH fixo - Capacitação dos Profissionais de Atendimento PréHospitalar Móvel - Rede Samu 192 e Pré-Hospitalar Fixo (http://www. capacitacaosamuhaoc. com.br/samu/login/ arquivos/apostila_SAMU_ v4.pdf, 2014). 
Trauma do Hospital. Segundo metade deles, a equipe seria suficiente numericamente e compatível com as necessidades do serviço, mas nenhum teceu comentários. Entretanto, a outra metade discorda, conforme relatos a seguir: "Não, porque nem todos estão preparados e são residentes sem a presença dos STAFFS" (P4). "Poucos profissionais" (P6). "[Multiprofissional], porém, não é compatível" (P9).

A percepção de como acontece o trabalho difere entre os participantes, porque a intenção, o sentir e os significados são produzidos de acordo com a relação singular que cada um estabelece com o objeto de trabalho.

De acordo com recomendações do Ministério da Saúde (BRASIL, 2011B, P. 3), um dos critérios de qualificação do pronto-socorro é "a organização do trabalho das equipes multiprofissionais de forma horizontal, em regime conhecido como 'diarista', utilizando-se prontuário único compartilhado por toda a equipe". No Hospital estudado, metade dos profissionais informou que atua em regime 'diarista' (que atua todos os dias em um ou dois turnos), enquanto a outra metade no regime 'plantão' (de 12 ou 24 horas).

Os gestores de saúde informaram unanimemente que há dificuldade na contratação de trabalhadores para o pronto-socorro: "Demora no concurso. Em caso de contratos, não contratam suficientes [referindo-se ao gestor responsável pela contratação dos profissionais de saúde]" (G1). "Falta de recursos humanos de enfermagem e treinamento" (G2). "Burocracia [quanto à contratação de recursos humanos]" (G3).

Ainda no que diz respeito à organização das equipes e ao trabalho prestado no serviço de pronto-socorro do Hospital, os gestores de saúde consideram: "Tumultuado, não sendo feito como deveria ser; cuidadores exaustos" (G1). "Dificil trabalhar com baixos recursos humanos e superlotação" (G2).

A qualificação dos trabalhadores que atuam nas urgências e emergências é fundamental para garantir a prestação efetiva de cuidados. Nesse sentido, seriam necessários investimentos sob a responsabilidade das três esferas governamentais.

A grande demanda de usuários e as dificuldades na organização dos fluxos assistenciais são problemas nevrálgicos para o Hospital: "Não há regulação de leitos para referenciar os pacientes. Pacientes mal orientados procuram o serviço sem necessidade. Entorno não faz contato prévio" (G1). "Dificuldade em efetivar a referência e a contrarreferência" (G2).

A demanda pelos serviços de saúde pode ser entendida como um pedido explicito que expressa todas as necessidades do usuário, podendo ser efetivada por meio de consulta, acesso a exames, consumo de medicamentos e realização de procedimentos, pois essa é a forma como os serviços organizam sua oferta. Paradoxalmente, as necessidades dos usuários podem ser outras, como respostas às questões socioeconômicas, às más condições de vida, à violência, à solidão, à necessidade de vínculo com um serviço/profissional, ou, ainda, o acesso a uma tecnologia específica que possa lhes proporcionar qualidade de vida (MARQUES; LIMA, 2007).

Altas taxas de internação por condições sensíveis à atenção primária em uma população, ou subgrupo(s) desta, podem indicar sérios problemas de acesso ao sistema de saúde ou de seu desempenho. O excesso de hospitalizações representa um sinal de alerta, que pode estar relacionado a problemas na rede de atenção, sobretudo na resolubilidade da atenção primária. 


\section{Conclusões}

O Hospital estudado se apresenta como um ponto de atenção estratégico para o SUS e enfrenta diversos problemas para se inserir na rede de atenção. Os resultados sugerem que:

- A definição de pactos e fluxos assistenciais incipientes não possibilita ao Hospital assumir o seu papel terciário e de "porta de entrada' para todo e qualquer paciente (referenciados e demanda espontânea), especialmente os que não são absorvidos e ordenados pela rede de atenção à saúde;

- Há problemas no funcionamento dos demais serviços de saúde, que, em geral, não promovem a seleção de casos clinicamente menos graves e intermediários e, consequentemente, não encaminham ao Hospital apenas pacientes clinicamente agudos ou graves, sendo essa a missão desse Hospital na rede de cuidados;

- A regulação do acesso é incipiente;

\section{Referências}

BRASIL. Ministério da Saúde. Banco de dados do Sistema Único de Saúde -DATASUS. Disponível em: $<$ http://www.cnes.datasus.gov.br>. Acesso em: 1 out. 2014a.

Ministério da Saúde. Banco de dados do Sistema Único de Saúde - DATASUS. Disponível em: <http:// www.tabnet.datasus.gov.br $>$. Acesso em: 3 out. 2014b.

Ministério da Saúde. Portaria n ${ }^{\circ} 1.600$, de 7

de julho de 2011. Reformula a Política Nacional de
- A Atenção Primária à Saúde da região onde se situa o Hospital é pouco resolutiva;

- Os gestores são ativos no processo de implantação da rede, porém, ainda é preciso aproximar os trabalhadores desse processo;

- Os profissionais de saúde estão em número insuficiente e com baixo protagonismo na implantação da RUE;

- Pouco investimento na educação permanente em saúde dos gestores e trabalhadores.

Com relação aos critérios de qualificação do serviço de pronto-socorro estabelecidos pelo Ministério da Saúde, há evidências de boas práticas quanto à articulação do Samu (pré-hospitalar) e da porta de entrada hospitalar de urgência e emergência.

Conclui-se que a inserção do Hospital na RUE acontece de forma lenta e dependente da articulação dos vários níveis de gestão do SUS.
Atenção às Urgências e institui a Rede de Atenção às Urgências no Sistema Único de Saúde (SUS). Diário Oficial [da] União, Brasília, DF, 8 jul. 2011a, p. 70.

Ministério da Saúde. Portaria n ${ }^{\circ} 4.279$, de 30 de dezembro de 2010. Estabelece diretrizes para a organização da Rede de Atenção à Saúde no âmbito do Sistema Único de Saúde. Diário Oficial [da] União, Brasília, DF, 31 dez. 2010, p. 88.

Portaria GM/MS no 2.395, de 11 de outubro de 
2011. Organiza o Componente Hospitalar da Rede de Atenção às Urgências no âmbito do Sistema Único de Saúde (SUS). Diário Oficial [da] União, Brasília, DF, 13 out. 2011b, p. 79-82.

. Resolução do Conselho Federal de Medicina $\mathrm{n}^{\circ} 1451$, de 10 de março de 1995. Resolve sobre estabelecimentos de Prontos-Socorros Públicos e Privados, equipe médica, sala de emergência, recursos técnicos mínimos disponíveis, funcionamento e porte destes estabelecimentos. Diário Oficial [da] União, Brasília, DF, 17 mar. 1995, p. 3666.

Ministério da Saúde. Secretaria de Atenção à Saúde. Departamento de Atenção Especializada. Manual instrutivo da Rede de Atenção às Urgências e Emergências no Sistema Único de Saúde (SUS). Brasília, DF: Editora do Ministério da Saúde, 2013.

CECILIO L. C. O.; MERHY E. E. A integralidade do cuidado como eixo da gestão hospitalar. In: PINHEIRO, R.; MATTOS, R. A. (Org.). Construção da integralidade: cotidiano, saberes e práticas em saúde. Rio de Janeiro: IMS: UERJ: ABRASCO, 2003, p. 197-210.

DEMORI, R.; PIZZOLATO, S. I. Acolhimento como perspectiva de reestruturação das emergências. 2007. 41 f. Trabalho de Conclusão de curso (Especialização em Gestão Hospitalar) - Grupo Hospitalar Conceição, Escola Nacional de Saúde Pública, Fundação Oswaldo Cruz, Rio de Janeiro, 2007.

DUBEUX, L. S.; FREESE, E.; FELISBERTO, E. Acesso a hospitais regionais de urgência e emergência: abordagem aos usuários para avaliação do itinerário e dos obstáculos aos serviços de saúde. Physis, Rio de Janeiro, v. 23, n. 2, p. 345-369, 2013.

FELICIANO, K. V. O.; KOVACS, M. H.; SARINHO, S. W. Sentimentos de profissionais dos serviços de pronto-socorro pediátrico: reflexões sobre o burnout. Revista Brasileira de Saúde Materno Infantil, Recife, v. 5, n. 3, p. 319-328, 2005.

\section{MACHADO, C. V.; SALVADOR, F. G. F.; O’DWYER, G.}

Serviço de Atendimento Móvel de Urgência: análise da política brasileira. Revista Saúde Pública, São Paulo, v.
45, n. 3, jun. 2011.

MAIO, M. C.; LIMA, N. T. Fórum: o desafio SUS: 20 anos do Sistema Único de Saúde. Introdução. Cadernos de Saúde Pública, Rio de Janeiro, v. 25, n. 7, p. 1611-1613, 2009.

MARQUES, G. Q.; LIMA, M. A. D. S. Demandas de usuários a um serviço de pronto atendimento e seu acolhimento ao sistema de saúde. Revista Latino-Americana de Enfermagem, Rio de Janeiro, v. 15, n. 1, p. 13-19, 2007.

MENDES, A. C. G. et al. Condições e motivações para o trabalho de enfermeiros e médicos em serviços de emergência de alta complexidade. Revista brasileira de enfermagem, Brasília, v. 66, n. 2, p. 161-166, 2013.

MINAYO, M. C. S. Pesquisa social: teoria, método e criatividade. 27. ed. Petrópolis: Vozes, 2008.

O’DWYER, G. A gestão da atenção às urgências e o protagonismo federal. Ciência \&t saúde coletiva, Rio de Janeiro, v. 15, n. 5, p. 2395-2404, 2010.

PEDUZZI, M. Equipe multiprofissional de saúde: conceito e tipologia. Revista de Saúde Pública, São Paulo, v. 35 , n. 1, fev. 2001.

SÁ, M. C.; CARRETEIRO, T. C.; FERNANDES, M. I. A. Limites do cuidado: representações e processos inconscientes sobre a população na porta de entrada de um hospital de emergência. Cadernos de Saúde Pública, Rio de Janeiro, v. 24, n. 6, p. 1334-1343, 2008.

SOUZA, R. B.; SILVA, M. J. P.; NORI, A. ProntoSocorro: uma visão sobre a interação entre profissionais de enfermagem e pacientes. Revista Gaúcha de Enfermagem, Porto Alegre v. 28, n. 2, p. 242-249, 2007.

Recebido para publicação em março de 2015

Versão final em junho de 2015

Conflito de interesse: inexistente

Suporte financeiro: não houve 